NAMES, Vol. 66, No. 4, December 2018, 233-245

\title{
Recipe Names as a Gateway to Interpersonal Communication
}

\author{
NATSUKO TSUJIMURA \\ Department of East Asian Languages and Cultures, Indiana University, \\ Bloomington, USA
}

An examination of Japanese cookbooks from the early twentieth century to date demonstrates that changes in the nature of recipe names over the past 87 years have made the relationship between the writer and the audience closer. Recipe names have come to be more informative and creative through the rich use of mimetics, metaphors, and word play. Furthermore, they have become more personalized and stylized. The personal stories behind these recipe names help the author elicit in readers a sense of camaraderie and/ or nostalgic memories that specific food items in a given historical period evoke. This article demonstrates two things: what seem to be simple names for recipes can be regarded as a gateway to interpersonal communication between the author and audience; and recipe names have evolved into an increasingly enriched form of communication over time by ingenious use of various tools available in the language.

KEYWORDS Recipe names, informativeness, brevity, interpersonal communication, identity, personalization

\section{Introduction}

Cookbooks and recipes are first and foremost about food, and their essential purpose is to give instructions on how to prepare dishes. But in addition to such an obvious practical function, they have been regarded as an independent genre of writing for scholarly analysis. Food memoirs, for one, have been subject to a wide range of investigation in disciplinary areas including anthropology, history, literature, sociology, and geography. This rich research application notwithstanding, often taken for granted is the importance of language and its linguistic contribution to inter- and multidisciplinary issues revolving around food and food writing (Jurafsky 20I4; Lehrer 2009). Recipes themselves often are embedded in food memoirs and other forms of primary sources of research within the genre of food writing, but they are of great importance and relevance to the study 
of language in their own right. Since the transmission of cooking instructions is made possible through language in social interaction (Cotter 1997), several linguistic investigations have already led to intriguing analyses, whether their orientation be quantitative, discourse analytic, or sociocultural, as the range is demonstrated in works such as those by Culy (1996), Fisher (1983), and Lakoff (2006).

Although there is a certain degree of disparity among authors, recipes are generally structured into several integral parts. Whitman and Simon (I993), for instance, list the following elements relevant to writing a recipe although not all of them are always included: title, headnote, ingredients list, instructions, servings line, note, and variation. Of these essential components, it seems palpable that the most indispensable parts of recipes are titles, lists of ingredients, and instructions.

Recipe titles will be focused on in this article as names of the dishes to be created. Surveying recipe titles that appear in Japanese cookbooks for the 87 years between I927 and 20I4 suggests that recipe names have undergone notable changes since the middle of the twentieth century. It is the goal of this article to demonstrate that these changes over time bear sociolinguistic and sociocultural implications. First, the degree of informativeness has been enhanced by the rich use of mimetics (i.e. onomatopoeia), metaphors, and word play. Second, recipe names have become more personalized and stylized, thereby drawing more attention and shortening the distance between the author and the reader. Humorous and enigmatic names that are demystified by the author's personal anecdotes exemplify this phenomenon.

\section{Background}

Recipes are a type of register that give concise instructions of how to prepare dishes, and share some practical aims with other registers like menus (Zwicky and Zwicky I980). Recipe names should serve as instant panoramic summaries of what given recipes are about, calling for a compressed set of informativeness and brevity. To give a brief illustration of recipe names in English, titles in (I) below, all from Garten's I999 cookbook, introduce recipes by the combination of primary and distinguishing ingredients and cooking methods such as roasting, grilling, and baking.

(I) Swordfish with tomatoes and capers (I36)

Roasted baby pumpkins (I 53 )

Grilled lemon chicken (48)

Beets with orange vinaigrette (93)

Baked Virginia ham (II9)

Recipe names can be further elaborated on by including additional ingredients like sauces - sometime in small captions or supplements as shown in (2a). Details may also be given by adjectives that describe the characteristic tastes, texture, temperature, and the speed of cooking. Examples are given in (2b), all taken from Lee (20I3).

(2) a. Cola ham hocks - with miso glaze (I I4)

Piggy burgers - with sun-dried tomato ketchup (II8)

Steak tartare - with a six-minute egg and strawberry ketchup (48)

b. Spicy napa kimchi (I65)

Warm shrimp salad - with lemongrass crumbs (I38)

Quick caraway pickles (I73) 
Examples in (3) - found in the local newspaper, The Herald Times (April I3, 2016, $\mathrm{D}_{7}$ ) - show that common, generic recipe names like oatmeal and salad may be detailed by explaining how each recipe stands out as something no longer ordinary. Or, recipe names can be featured by trendy cooking methods like slow cooking or healthy and sustainable eating like vegetarianism. Such information is welcomed by those following a special diet, whether due to one's beliefs surrounding foodways or for health reasons. Increasing details and their nature reflect narrower and more precise branching of personal choices for what and how people consume in recent years.

(3) Midmorning oatmeal

Make-ahead yogurt parfaits

Overnight slow cooker oatmeal

Vegan Caesar salad [Note: no eggs, no anchovies]

While the focus of the information varies, these recipe names attempt to be as informative and brief as possible.

The analysis given in this article is based on a survey of 22 Japanese cookbooks that were published in the genre of home cooking between I927 and 20I4. I have chosen the I927 cookbook as the oldest source since deciphering accessible publications before that time requires special linguistic skills. Note also that war and its aftermath may explain the dearth of available published cookbooks in the I940s.

\section{Enrichment of informativeness in recipe names}

The most typical patterns of Japanese recipe names in the early to mid-twentieth century take either of the morphological forms in (4). The particle -no in (4a) is the genitive case marker; the names shown in $(4 \mathrm{~b})$ take the compound form:

(4) a. <ingredients> -no <cooking method/style>

\begin{tabular}{|c|c|c|}
\hline & yamakake & "yellowtail topped with grated yam" \\
\hline yellowtail-gen & grated-yam-topping & (NHK I927) \\
\hline yasai-no & korokke & "vegetable croquette" \\
\hline vegetable-gen & croquette & (NHK I935) \\
\hline goboo-no & yawaraka-ni & "tender-cooked burdock" \\
\hline burdock-gen & tender-cooked & (Egawa I957) \\
\hline & cooking method & $\mathrm{e}>$ or $<$ cooking $\mathrm{m}$ \\
\hline chil & "chic & en curry" \\
\hline rry & & $(\mathrm{NH}$ \\
\hline -gayu & "ric & oorridge with seven plants" \\
\hline th-ric & & (NHK I927) \\
\hline j-soomen & "chill & d somen noodles" \\
\hline & & (NHK I935 \\
\hline
\end{tabular}

These recipe names minimally satisfy the goal of informativeness and brevity by referring to major ingredients and the manner of cooking or preparation. Although the degree of informativeness may be comparatively meager, the minimum information of ingredients and style of cooking is sufficiently provided in a few words. 
While the patterns in (4) remain as prevalent forms for recipe naming throughout the historical span under the current survey, recipe names have come to be more detailed since the r 980 s. One way of increasing informativeness is the use of mimetics, which serve to provide multiple dimensions of descriptions. Mimetic words in recipe names can describe not only the expected taste but also the texture of the finished dish or the sound and manner of the cooking process. The mimetic vocabulary appeals to the five senses - vision (appearance), hearing (sound), smell, taste, and touch (texture) - and offers a succinct portrayal of a recipe. Thus, mimetics are an economical linguistic means for simultaneously achieving a higher level of informativeness and brevity.

The two patterns in (5-6) are common in recipe names with mimetics. In (5) the first noun that refers to an ingredient modifies the following mimetic word, mediated by the genitive marker - no. The recipe names in (6) are a juxtaposition of a mimetic word and a noun that refer to the primary ingredient or the cooking style (or category) of an intended dish, such as salad, cooking in a soupy broth, and stir/deep-frying. Those in (7) demonstrate the combination of the two patterns in $(5-6)$. To the right of each example is indicated in square brackets the specific sense to which the bold-faced mimetic word is intended to appeal.

(5) <ingredients >-no <mimetic >
a. kyabetsu-no shin-no huwahuwa [texture]
cabbage-gen core-gen fluffiness
"fluffy cabbage core" (Egawa I957)
b. shiozake-no juQ [sound] ([Q]=glottal stop)
salted.salmon-gen sizzling
"sizzling salted salmon" (Kobayashi I995)
c. piiman-no kutakuta [texture]
green.pepper-gen wilted/softened
"wilted/softened green peppers" (Kobayashi 2007)
d. kyuuri-no gorogoro [appearance]
cucumber-gen cubes
"cucumber cubes" (Kobayashi 2007)

(6) <mimetic $>$-<ingredients/style>

a. purupuru-konnyaku [texture/appearance]

elastic.and.wobbly-konnyaku

"elastic and wobbly konnyaku" (Y. Tatsumi I978)

b. huwahuwa-tamago [texture]

fluffy-eggs

"fluffy eggs" (Y. Tatsumi I978)

c. sappari-poteto-sarada [taste]

light-potato-salad

"light potato salad" (Kidosaki I987)

d. pokkapoka-suupuni [feel (body temperature)]

warm-cooking.in.soup

"cooked in soup (which keeps you warm after eating it)" (Kobayashi 2007)

e. paripari-kitsune [sound/texture]

crispy-fox

"crispy deep-fried tofu” (Kobayashi I995) 


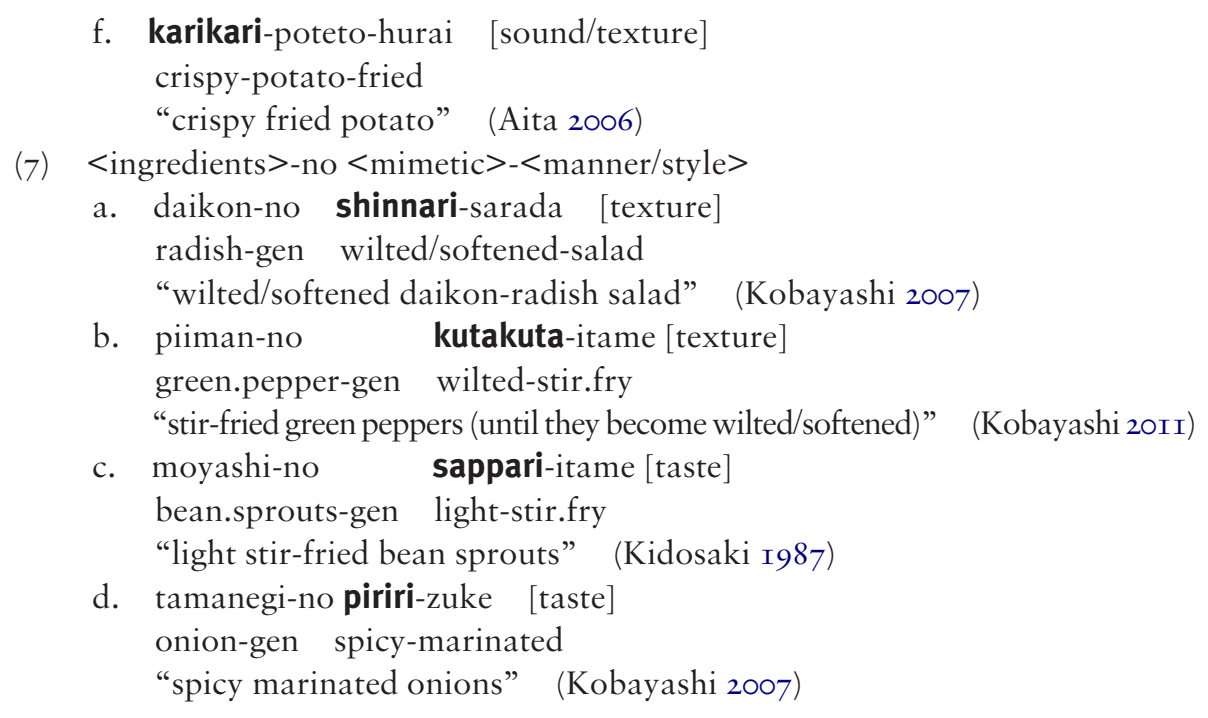

Descriptions that mimetics facilitate range from texture (touch), shape of ingredients and overall appearance (vision), taste, to a specific feeling in the mouth and a warm body temperature after eating a prepared dish. For example, huwahuwa in (5a) and (6b) captures the soft and fluffy texture of a finished dish as its ideal state. The name in ( $5 \mathrm{a}$ ) suggests that cabbage cores that are frequently discarded can find a good culinary use and that the dish promises an unexpected texture for easy digestion.

Sound-based mimetics like those in (5b), (6e), and (6f) have a vivid appeal; additionally, (6e) and (6f) have cross-modal references to texture as well as sound. In ( $5 \mathrm{~b}), j u \mathrm{Q}$ symbolizes the sizzling sound made when salmon is pan-fried. In fact, the mimetic word contains richer information than the sound itself: the word $j u Q$ suggests the use of a little oil as an ingredient and high heat as a cooking method. A single mimetic vocabulary item can thus provide elaboration on various aspects of food preparation that may not otherwise be achieved by non-mimetic counterparts. The two mimetics in (6e) and (6f), paripari and karikari, are also sound-based, both symbolizing the expected sounds upon biting - a sound similar to the one made when eating potato chips and raw vegetables like carrots and cucumbers. Biting and chewing sounds are often associated with the texture of the foods, and paripari and karikari in (6e) and (6f) indeed achieve auditory and tactile appeals simultaneously.

The mimetics in $(5 \mathrm{~d})$ and $(6 \mathrm{a})$ feature the appearance of the ingredients by way of vision-based symbolism. The general meanings relevant to gorogoro in $(5 \mathrm{~d})$ are (i) "the manner of a round or cylindrical object or objects rolling along" and (ii) "the state in which things exist or lie about in abundance" (Kakehi, Tamori, and Schourup I996, 433434). Typical objects described under the sense in (i) include logs and rocks. Gorogoro in $(5 \mathrm{~d})$ obviously does not refer to any dynamic movement, but the combination of the two senses in (i) and (ii) gives rise to a visual image of many bite-sized (round or cubic) cucumbers. This image helps the reader instantly picture the process of preparing cucumbers, i.e. being cut into bite-size chunks rather than sliced or finely chopped. Purupuru in (6a) generally depicts the manner in which an elastic or springy object quivers; food-related examples to fit the description include Jello and pudding. Associated with the quivering motion is the elastic texture, so purupuru carries information regarding the 
visual and tactile properties of the ingredient. Konnyaku is considered to have virtually no taste, but the use of purupuru suggests that the recipe capitalizes on the positive texture quality of konnyaku, making the expected dish more attractive.

The mimetic words in $(6 \mathrm{c}),(7 \mathrm{c})$ and $(7 \mathrm{~d})$ emphasize the taste more directly than others. The mimetic modifier sappari for potato salad in (6c) and stir-fried bean sprouts in $(7 \mathrm{c})$ not only suggests a lighter taste, perhaps with less or no fatty ingredients, but also gives a sensual effect leading to a refreshing mouthfeel that standard cooking methods or ingredients may not bring out. As a typical descriptor for spiciness and even for a sharp pain, piriri in $(7 \mathrm{~d})$ immediately evokes the stinging sensation of onions and spices like red pepper flakes. Finally, pokkapoka in (6d) describes the warm body temperature that will result from eating the soupy dish. In looking for a recipe during the cold winter season, soups and soupy dishes may be ideal, but the addition of the single mimetic word pokkapoka to a recipe name has an even more vivid impact on the reader's process of selecting recipes.

As the examples in $(5-7)$ illustrate, the mimetic vocabulary is used to give a concise summary of a dish that is informative enough to draw the reader's attention. While detailing the essential ingredients and the style or category of cooking, mimetic words also can elicit in the reader's mind subjective experiences of eating or cooking the food, however imaginary it may be. Furthermore, the sensual appeals of mimetics are often cross-modal, impacting more than one domain of our senses. A single mimetic expression in a recipe name, can thus speak to - and promote - multiple qualities of the recipe vividly and instantly.

In earlier cookbooks especially before I960, sporadic appearances of mimetic words are detected but are limited to only a few recurring terms: for instance, huwahuwa, which refers to the fluffy texture of eggs, and paripari, which describes the crunchy and crisp texture of fresh vegetables. The use of mimetics varies depending on the author, but generally the last two decades of the twentieth century mark the beginning of their higher frequency both in token and type counts, and the inclusion of mimetics in recipe names has no longer been considered novel. Furthermore, as mimetics routinely appear in recipe names, their altered forms often emerged. The mimetics in (8) are slightly different from their conventional forms, and signal a trend of rising colloquialism.

(8) a. purippuri ebi-chiri-don (<puripuri)

springy shrimp-spicy-rice.bowl

"spicy springy shrimp in a rice bowl" (Okada 2007)

b. mocchimochi kaisen chijimi (<mochimochi)

chewy sea.food Chijimi

"chewy seafood Chijimi [savory Korean pancake]" (Okada 2007)

c. toro ri hanjuku oyako-don (<torori)

meltingly.soft half.boiled parent.child-rice.bowl

"rice bowl with the topping of chicken and melting half-boiled egg" (Aita 2006)

The mimetic words purippuri and mocchimochi in (8a-b) are considered emphatic forms of puripuri and mochimochi, respectively, with long (or geminate) consonants. These emphatic variants occur more frequently in speech. The combination of the emphatic forms and the speech-oriented style leads to casualness in recipe naming. In 
(8c) the base word [torori] is pronounced as [toroori] with the second vowel lengthened for emphasis. This recipe name is additionally interesting from the orthographical standpoint. Vowel lengthening is customarily represented by a horizontal bar, as in $と$ ろーり, but replacing it with a tilde as in とろ〜り invites a visual image that creamy egg yolk warmly oozes out.

Another way of increasing informativeness is found in the type of information included in recipe names that was not previously part of them. In addition to ingredients and cooking styles, cooking utensils like (9a-b) and appliances as in (9c) serve as a new type of information:

(9) a. huraipan-sukiyaki

frying.pan-sukiyaki

"sukiyaki in a frying pan" (Okada 2007)

b. sara-udon

plate-udon

“udon served on a plate” (Kobayashi I995)

c. toosutaa-de kantan tebaniku

toaster-by easy chicken.wing

“easy chicken wing in an (oven) toaster" (Okada 2007)

While sukiyaki is commonly prepared in a hot pot for table-top cooking, a frying pan is substituted for it in (9a), suggesting a somewhat easier stove-top preparation. Recipe names with $u d o n$ often assume noodles with soup in a deep bowl. The dish named by (9b), instead, is served on a plate. The reference to sara "a (flat) plate" as serving-ware further gives a clue that the noodle is stir-fried or simply mixed. The oven toaster in the recipe name in $(9 c)$ indicates the reliance on more modernized cooking appliances that most homes now have, while also implying a less fastidious cooking procedure and reduced cooking time. Thus, the incorporation of cooking utensils, serving-ware, and appliances can implicitly offer further details about recipes before reading cooking instructions.

Also included are unique features that showcase the recipes as in (IO):

(IO)
a. hoorensoo-to moyashi-no
booshi-nokke
spinach-and bean.sprouts-gen hat-placing
"spinach and bean sprouts topped with a hat [eggs]" (Kurihara I992)
b. shomin-no
ebi-chaahan
common.people-gen shrimp-fried.rice
"shrimp fried rice for common people" (Kobayashi 2007)
c. gakubuchi toosuto
picture.frame toast
"picture-frame toast” (Deguchi 2002)

In the recipe for (Ioa), stir-fried spinach and bean sprouts have an additional topping of cooked eggs, a novel way of serving common stir-fried dishes. Through the metaphorical use of a hat for eggs as a topping, the reader can visualize an alternative to mundane presentation styles. The name for stir-fried rice in (Iob) has the modifier shomin-no "for the common people" that may offer some solace from economic concerns 
stemming from the high cost of shrimp. The cost-effective ingredient in lieu of fresh shrimp is a dried version called sakura-ebi "dried sakura shrimp", at least at the time when the recipe was written in 2007. The name "picture-frame toast" in (IOc) describes the shape of the resulting dish by using a visual metaphor.

These examples of names clearly provide more detailed information about given recipes, highlighting their distinctive or appealing aspects as they are relevant to various dimensions of cooking and taste. Informativeness is undeniably motivated by consumerism, and tactful use of language accommodates an effective vehicle to achieve that.

\section{Personalization and stylization of recipe names}

With the increasing degree of informativeness over time, recipe names also have become more personalized and stylized, shortening the distance between the author and the reader. This phenomenon is reflected by witty, entertaining, and sometimes enigmatic names that are demystified by personal anecdotes provided in the authors' commentaries. Our earlier examples in (Io) illustrate this aspect, often eliciting humorous reactions or heart-warming feelings. Additional examples are given in (II):

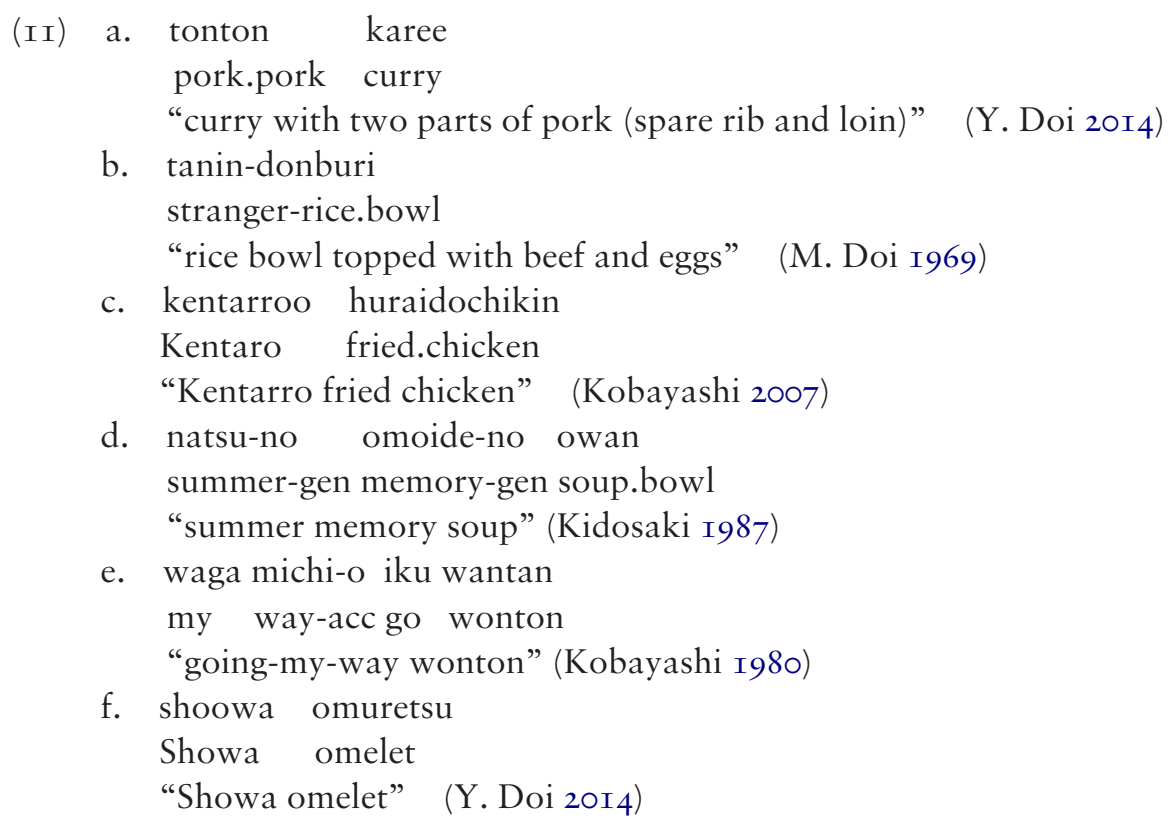

The single syllable ton of tonton in (I Ia) stands for pork, as in ton-katsu "pork cutlet" and ton-jiru "pork soup", but repeating the syllable as tonton makes an iconic reference to two pork parts, spare rib and loin in this case. The recipe name in (IIb) reminds the reader of a common dish, oyako-donburi "parent.child-rice.bowl", which consists of chicken (parent) and eggs (child). In ( $\mathrm{I}$ b), however, beef is substituted for chicken, and thus beef and eggs are comically characterized as "strangers" contrastive of the parent-child relation of chicken and eggs. The humorous and uncommon naming anticipates special ingredients. 
The name in (IIC) sounds like Kentucky Fried Chicken. Kentaroo is the name of the author's son, and fried chicken is his favorite food. Interestingly, the sound $/ \mathrm{r} /$ in Japanese is never lengthened (geminated), so [kentarroo] of this recipe name is not only difficult to pronounce but it is linguistically an anomaly. However, breaking the linguistic norm in the use of [kentarroo] shows the author's wit and skill to parallel it to the Japanese pronunciation of Kentucky: [kentakkii] and [kentarroo]. Underlying this inventive naming is the mother's love in making her son's favorite dish. The name in (IId) bears a poetic tone with a somewhat pretentious air. The author reminisces a summer vacation sitein lieu of heat and humidity - setting the stage for the vegetable-rich soup she made. In her commentary, she playfully admits the affectation that the name natsu-no omoide "summer memory" may have but quickly gives herself permission for the rare pretention.

The author of (IIe) remembers that the dish was created by accident. Wonton is made with meat filling, but she ran out of time to make a standard wonton soup. Instead of forming the ingredients into the customary dumpling shape, she ended up throwing each ingredient of wonton wrappers and meat separately into a soup broth. The recipe name concisely captures the unorthodox cooking process. Furthermore, the use of metaphor in the name allows for personification of the ingredients, adding a humorous touch: wonton wrappers and meat filling are treated as if they had their own mind, separately jumping into the soup instead of the meat filling inside the wrappers. This accidental dish turned out to be surprisingly delicious and additionally time-saving although unintentionally so. An underlying message is that the author is a busy woman herself and cooking for her family does not always, and more importantly need not, produce something perfect.

Finally, the name in (IIf) has the effect of sharing in the nostalgic taste of the Showa period (I926-89). Although Western-style dishes (e.g. omelet, pork cutlet, and curry) already had been introduced during the Meiji period (I868-I9I2), they were adapted to Japanese home cooking especially after World War II. The omelet that was widely known during the Showa era was made with a filling of sauteed ground meat and onions. Reference to the Showa era in recipe names has a special appeal to a particular age group, especially the baby boomers. Food tastes are intricately linked to our memories, and the name shoowa omuretsu in (IIf) certainly takes the audience on such a sentimental journey.

Overall, the sample names in (II) may not be overly successful regarding informativeness, but nevertheless they embed a number of personal anecdotes to be shared with the reader. This is an important role of recipe names, i.e. evoking past memories, when we view recipes as a venue of food discourse. All the recipe names in (II) and many more like them have appeared since the mid-twentieth century to date, and they are crucially different from the earlier samples of recipe names in displaying, explicitly or implicitly, the personal side of the author. The new style in recipe names allows the author to communicate with the reader at a significantly closer distance than before. That is, these names play a role as a gateway to building identity between the two parties by calling to mind a sense of camaraderie.

The closer distance between the author and the reader in recipe names is achieved even more directly at the turn of this century, especially as the use of social media becomes more prevalent. Although recipes and recipe names that appear on social networks are not within the scope of the current study, we briefly examine cookbooks that compiled recipes on various media venues including TV shows and blog sites. In contrast with - or 
in conjunction with - the rich use of linguistic and rhetorical means observed earlier, colloquialisms are becoming widespread in recipes, and accordingly recipe names are affected by the shift of style in language. Since transmission of cooking instructions through Internet-based communication media is increasingly popular among younger people, the colloquialism arguably reflects the communication style of the younger generation and appeals to them as a primary target audience. Also, the medium itself promotes more informal language use. Earlier, we have witnessed in (8c) that the lengthened vowel in toro ri reflects the speaking style, and that the colloquialism is staged further by the visual effect of the tilde. The marriage between colloquial speech and orthographic tools is readily found in cookbooks published in the twenty-first century. To begin, the tilde has become one of the most frequent symbols in recipe names as shown in (I2):

(I2) a. SPAM raisu de su. [SPAMライスで〜す] SPAM rice is "It's SPAM rice." (Aita 2006)

b. Nattoo-pasuta de〜 su. [納豆パスタで〜〜す] fermented.soy.beans-pasta is "It's pasta with Natto." (Aita 2006)

Both recipe names in (I2) use the tilde, and even twice in (I 2 b). It is additionally interesting that these names have the sentential ending, desu "(it) is", even though recipe names customarily use the noun phrase form as shown in previous examples. The combination of the sentential ending, the elongated vowel to mark a colloquialism, and the tilde makes one feel as if the author were speaking casually to the (young) audience. Note that (I2a) keeps the name of the ingredient, SPAM, in English. The orthographical mixing of the two languages and the use of the American ingredient together give rise to an appeal to younger users. The casual communication style as well as modernity and globalism represented by the foreign ingredient help build generational and cultural identity between the author and the audience.

Other symbols like the asterisk and the exclamation point as shown in (I3) are growing common as well:

(I3) a. huraipan hitotsu-de kantan*oyako-don [フライパンひとつで簡 単 * 親子井]

frying.pan one-by easy*parent.child-rice.bowl

"easy rice bowl with chicken and egg topping, made with only a frying pan”

(Okada 2007)

b. daininki-no GBS-o gyuuniku-de!! [大人気のGBSを牛肉 で! ! ]

very.popular-gen GBS-acc beef-with!!

"popular beef with garlic, butter, and soy sauce" (Aita 2006)

The function of the asterisk in ( $13 \mathrm{a}$ ) is not transparent but seems to ease the flow from the first part onto the essential part of the name, oyako-don, without verbal elaboration. The repeated exclamation mark in ( 13 b) serves to exude the excitement of introducing 
a recipe with the very popular GBS i.e. a culinary concoction of garlic, butter, and soy sauce (Note: GBS=garlic, butter, and shooyu "soy sauce").

Finally, (I4) presents an almost extreme use of the spoken style by naming a dish with the firmly colloquial phrase, nanchatte "just kidding":

(I4) kinoko ippai-no nanchatte rizotto

"risotto with a lot of - just kidding - mushrooms" (Aita 2006)

Viewed from the perspective of Grice's (1975) maxim of quality - what is said should not be false in cooperative communication - the example at (I4) does not seem to be credible, especially given that the accuracy of the information is essential in instruction-giving discourse like recipes. The phrase nanchatte "just kidding", used only in a very casual and relaxed setting, overrides the communication norm, but the underlying humor serves as an agent to forgive the contradiction. Interestingly, while the recipe names in (I2-I4) use the colloquial style, approaching the audience more casually, they are not particularly brief. Some of the names could actually strike one as chatty and even wordy. Compared with the patterns surveyed earlier, then, we may be witnessing a more recent change in recipe names in social media where amusing and chatty rhetoric potentially plays a more significant role than brevity.

\section{Conclusion}

A recipe constitutes a complete narrative with communicative purposes (Cotter I997; Lakoff 2006). Serving as the recipe's "face" that the audience is most likely to look at first, a recipe name should contain as much relevant and helpful information about the dish in as few words as possible. At the same time, it should draw the reader's attention so that $\mathrm{s} /$ he decides to move onto the recipe itself. If, as we leaf through the cookbook, the recipe name remains as an ordinary word or phrase that is routinely heard in our daily life, the message that the author intends to convey in the recipe could be easily overlooked. Thus, it is critical for a recipe to strike an engaging outlook in its naming.

In this article, I have reported on changes in recipe names that appear in Japanese cookbooks published since the early twentieth century. Focusing on sociolinguistic and sociocultural roles that recipe names play in communication, two generalizations particularly have been singled out to capture the nature of the change. First, recipe names have increased informativeness through language-specific linguistic tools. We have observed that the mimetic vocabulary is efficient and effective due to their sensual references. Since a single mimetic word often can appeal to multiple human senses (e.g. vision-touch, sound-touch), it is a very resourceful linguistic means in showcasing the selling points of a recipe. It has also been demonstrated that greater informativeness can be achieved by creative manipulation of the language as is demonstrated by metaphors - including implied visual metaphors - and word play.

Second, from a sociocultural point of view, recipe names have become more personalized and stylized, so that they serve as an excellent introduction to the author's storytelling - sometimes very personal and relatable to the audience - as it is relevant to the recipe. Personal accounts offer an excellent venue for the authors to reach out to the audience by telling personal stories and revealing their private side. Although a full version of the 
story is usually provided by commentaries throughout the recipe, recipe names function as shorthand for the full version. As such, humorous, creative, and stylized names present a successful summary of what the audience can expect from the full version. The change in the degree of personalization in recipe names, mirroring richer personalization of recipes as a whole, has allowed the author to become closer to the audience.

Eating is such a fundamental need of humans, and the topic of food and eating seems to be a natural arena in which we allow ourselves to be open to others. Our examination of recipe names suggests that what seem to be simple labels for recipes can be viewed as a means of communication through which the author shares his or her identity with the audience. In addition to informativeness and brevity, then, Japanese recipe names, particularly in the late twentieth century to date, have come to achieve a significant role as a gateway to building a closer interpersonal relationship between the two parties regarding food, cooking, and beyond.

\title{
Acknowledgements
}

I would like to thank two anonymous referees for their helpful comments and suggestions that significantly improved the content of this article. Thanks also go to Stuart Davis for discussing various aspects of the larger project. Preliminary versions of this article were presented at the Annual Meeting of the American Name Society held in Austin, TX, on 5-8 January 2017, and the Annual Meeting of the Association for the Study of Food and Society held at Occidental College in Los Angeles on I4-I6 June 20I7. I thank the attendees of these conferences for their input.

\section{Disclosure statement}

No reported conflict of interest was reported by the author.

\section{Cookbooks consulted}

\author{
Aita, Koji. 2006. [I20-man-nin-no Oishii! Tsumatta] Koochan-no Kantan-ryoori Reshipi. Tokyo: Takarajima-sha. \\ Deguchi, Kazumi. 2002. Otoko-no Ryoori Kyooshitsu. Tokyo: Shunposha. \\ Doi, Masaru. I969. Wahuu Kyoo-no Ryoori. Tokyo: Nihon Hoso Shuppan Kyokai. \\ Doi, Yoshiharu. 20I4. Doi Yoshiharu-no Natsukashi Gohan. Tokyo: NHK Shuppan. \\ Egawa, Tomi. 1957. Watashitachi-no Okazu. Tokyo: Shibata Shoten. \\ Garten, Ina. 1999. The Barefoot Contessa Cookbook. New York: Clarkson Potter Publishers. \\ Kidosaki, Ai. 1986. Sutekina Kidosaki Ai no Osusume Ryoori. Tokyo: Gurahusha. \\ Kidosaki, Ai. 1987. Isogashii Hito-no Oishii Osoozai. Tokyo: Kairyusha. \\ Kobayashi, Katsuyo. 1980. Kobayashi Katsuyo-no Rakuraku Kukkingu. Tokyo: Bunka Shuppankyoku. \\ Kobayashi, Katsuyo. 1995. Kobayashi Katsuyo-no Zakkubaranni Omotenashi. Tokyo: Nihon Hoso Shuppan Kyokai. \\ Kobayashi, Katsuyo. 2007. Kobayashi Katsuyo-no Mainichi Okazu. Tokyo: Kodansha. \\ Kobayashi, Katsuyo. 20I . Kobayashi Katsuyo-no "Haha Okazu”. Tokyo: Kodansha. \\ Kokubun, Taichi, and Kentaroo Kobayashi. 2009. Taichi x Kentaroo Danshi Gohan-no Hon. Tokyo: M.Co. \\ Kurihara, Harumi. 1992. Gochisoosama-ga Kikitakute. Tokyo: Bunka Shuppankyoku. \\ Lee, Edward. 2013. Smoke \& Pickles: Recipes and Stories from a New Southern Kitchen. New York: Artisan. \\ NHK (Nihon Hoso Kyokai), eds. I927. Shiki no Ryoori. Tokyo: NHK. \\ NHK (Nihon Hoso Kyokai), eds. I928. Hibi no Ryoori. Tokyo: NHK. \\ NHK (Nihon Hoso Kyokai), eds. I935. Hooso Ryoori Issenshuu. Tokyo: NHK. \\ Nishi, Junichiro. 2007. Otoko no Reshipi. Tokyo: Gurahusha. \\ Okada, Shiori. 2007. Tsukutte Agetai Kare-gohan. Tokyo: Takarajima-sha.
}


Sawasaki, Umeko. I94I. Katei Ryoori Kiso-Hen. Tokyo: Hujinnotomosha.

Shiota, Maruo. I997. Shinshi-no Daidokoro. Tokyo: Gurahusha.

Tatsumi, Hamako. I960. Teshio ni Kaketa Watashi no Ryoori. Tokyo: Hujinnotomosha.

Tatsumi, Yoshiko. 1978. Yuzuriuketa Haha-no Aji. Tokyo: Hujinnotomosha.

The Herald Times. 2016. Bloomington, IN.

\section{References}

Cotter, Coleen. I997. "Claiming a Piece of the Pie: How the Language of Recipes Defines Community.” In Recipes for Reading: Community Cookbooks, Stories, Histories, edited by Ann L Bower, 5I-7I. Amherst: University of Massachusetts Press.

Culy, Christopher. 1996. "Null Objects in English Recipes." Language Variation and Change 8: 9I-I24.

Fisher, M. F. K. I983. With Bold Knife and Fork. London: Chatto \& Windus.

Grice, H. Paul. 1975. "Logic and Conversation." In Syntax and Semantics, vol. 3: Speech Act, edited by Peter Cole and Jerry Morgan, 43-58. New York: Academic Press.

Kakehi, Hisao, Ikuhiro Tamori, and Lawrence Schourup. 1996. Dictionary of Iconic Expressions in Japanese. Berlin: Mouton de Gruyter.

Jurafsky, Dan. 20I4. The Language of Food: A Linguist Reads the Menu. New York: W.W. Norton \& Company.

Lakoff, Robin. 2006. "Identity a la Carte: You are What You Eat.” In Discourse and Identity, edited by Anna de Fina, Deborah Schiffrin and Michael Bamberg, I42-I65. Cambridge: Cambridge University Press.

Lehrer, A. 2009. Wine \& Conversation. $2^{\text {nd }}$ ed. Oxford: Oxford University Press.

Whitman, Joan, and Dolores Simon. I993. Recipes into Type: A Handbook for Cookbook Writers and Editors. New York: HarperCollins Publishers.

Zwicky, Arnold, and Ann Zwicky. I980. "America's National Dish: The Style of Restaurant Menus." American Speech 55 (2): 83-92.

\section{Notes on contributor}

Natsuko Tsujimura is Professor of East Asian Languages and Cultures and Adjunct Professor of Linguistics at Indiana University, Bloomington. She is the author of $A n$ Introduction to Japanese Linguistics, 3 rd ed. (Wiley-Blackwell, 20I4), and the editor of Japanese Linguistics: Critical Concepts (Routledge, 2005) and The Handbook of Japanese Linguistics (Blackwell, I999). Her published articles in journals and book chapters have focused on a wide range of issues in Japanese linguistics relevant to topics in phonology, morphology, semantics, syntax, and sociolinguistics. She has served as Editor-in-Chief of Journal of Japanese Linguistics (I999-2005), and as Review Editor of Language (2009-2013).

Correspondence to: Natsuko Tsujimura, Department of East Asian Languages and Cultures, Indiana University, Global and International Studies Building, 355 N. Jordan Avenue, Bloomington, Indiana 47405-I105, USA. Email: tsujimur@indiana.edu 